\title{
Nonperturbative Twist in the Generation of Extreme-Ultraviolet Vortex Beams
}

\author{
Laura Rego, ${ }^{1, *}$ Julio San Román, ${ }^{1}$ Antonio Picón, ${ }^{2}$ Luis Plaja, ${ }^{1}$ and Carlos Hernández-García ${ }^{1, \dagger}$ \\ ${ }^{1}$ Grupo de Investigación en Aplicaciones del Láser y Fotónica, Departamento de Física Aplicada, \\ University of Salamanca, E-37008 Salamanca, Spain \\ ${ }^{2}$ Argonne National Laboratory, Argonne, Illinois 60439, USA
}

(Received 3 June 2016; published 14 October 2016)

\begin{abstract}
High-order harmonic generation (HHG) has been recently proven to produce extreme-ultraviolet (XUV) vortices from the nonlinear conversion of infrared twisted beams. Previous works have demonstrated a linear scaling law of the vortex charge with the harmonic order. We demonstrate that this simple law hides an unexpectedly rich scenario for the buildup of orbital angular momentum (OAM) due to the nonperturbative behavior of HHG. The complexity of these twisted XUV beams appears only when HHG is driven by nonpure vortex modes, where the XUV OAM content is dramatically increased. We explore the underlying mechanisms for this diversity and derive a general conservation rule for the nonperturbative OAM buildup. The simple scaling found in previous works corresponds to the collapse of this scenario for the particular case of pure (single-mode) OAM driving fields.
\end{abstract}

DOI: 10.1103/PhysRevLett.117.163202

High-order harmonic generation (HHG) [1-3] represents one of the most fascinating processes in strong-field physics, occurring during the interaction of intense lasers with matter. In contrast to low-order harmonic generation, HHG cannot be explained using perturbative physics. However, it can be understood with simple semiclassical arguments [4,5]: during the interaction with the laser pulse, a wave packet is tunnel ionized from an atom or molecule and then accelerated and driven back to the parent ion during the field oscillation. Upon recollision, the energy acquired from the laser field is released in the form of high frequency harmonics. The detected HHG radiation is subjected to the phase matching of the coherent emission from different radiators in the target $[6,7]$. In contrast to the perturbative case, where the intensity of subsequent harmonics of the driving field decays exponentially [8], the HHG spectrum presents a plateau of harmonics extending to high frequencies with nearly constant intensity. As a result, from the spectral point of view, HHG provides a unique source of radiation extending from the extreme ultraviolet (XUV) to the soft $\mathrm{x}$ rays $[9,10]$. Temporally, high harmonics can be synthesized into pulses of attosecond duration [11-14], a unique feature that has triggered a revolutionary metrological tool for the temporal characterization of ultrafast processes at the atomic and molecular scale [15-17].

The coherent nature of the HHG process conveys the possibility of mapping properties of the driving field into the shorter-wavelength radiation, thus providing a precise degree of control of the generated field through adequate

Published by the American Physical Society under the terms of the Creative Commons Attribution 3.0 License. Further distribution of this work must maintain attribution to the author(s) and the published article's title, journal citation, and DOI. modifications of the driver. In this context, the generation of XUV vortices, i.e., XUV beams carrying orbital angular momentum (OAM), has been recently reported using an infrared (IR) driver with OAM [18-20]. The Fourier synthesis of these XUV harmonic vortices results in the emission of helical attosecond beams with unprecedented spatiotemporal properties, predicted in Ref. [19] and recently observed in Ref. [21]. This promising scenario circumvents the limitations of other approaches that directly imprint OAM into the XUV field using spatial light modulators or holographic plates, whose efficiency is compromised at those wavelengths.

Optical vortices, i.e., twisted beams carrying OAM, add a supplementary degree of freedom to light-matter interactions [22-25], conveying additional information about the fundamental processes involved. For instance, it has been demonstrated that the dipole selection rules of singlephoton ionization must be extended to include the interplay between angular momentum and energy conservation [26]. Most strikingly, HHG driven by an OAM beam with a unique topological charge $\ell$, studied first theoretically [19] and later experimentally [20], leads to the simple scaling $\ell_{q}=q \ell\left(\ell_{q}\right.$ being the topological charge of the $q$ th harmonic). This simple rule follows the energy conservation of the harmonic conversion process $\omega_{q}=q \omega$ ( $\omega$ being the frequency of the fundamental), also found in perturbative harmonic generation [27-30]. However, this simple scaling, which is well understood as a consequence of the symmetry of the field, disguises the nonperturbative nature of HHG, where the number of photons involved in the process is not well defined [20].

In this Letter, we present a theoretical study of HHG driven by nonpure vortex beams, carrying different OAM contributions, to reveal the XUV OAM buildup due to the 
nonperturbative behavior of HHG. We perform advanced quantum simulations of OAM HHG and develop a simple model, the thin slab model, which allows us to obtain a clear insight of the underlying physics. We show that the use of nonpure vortex drivers increases dramatically the OAM content of the harmonics, mainly due to the nonperturbative intrinsic phase of the HHG process. We derive a general conservation rule for the OAM buildup in HHG that is more generic than the simple linear law governing perturbative harmonic generation and HHG driven by pure (single OAM mode) IR vortices. Our results are found very relevant for vortex drivers with only a fractional modal contamination and thus essential for the understanding of realistic OAM HHG experiments, where non-100\% pure vortices are used.

The physical scenario of our OAM HHG study is sketched in Fig. 1(a). An IR pulse, resulting from the superposition of two well-defined OAM beams [see Fig. 1(b)] of the same wavelength $\lambda_{0}$, is focused into an argon gas jet. Each atom interacting with the IR field emits XUV or soft x-ray radiation that is detected in the far field. The spatial structure of each vortex is represented as a LaguerreGaussian beam propagating in the $z$ direction and expressed as $\mathrm{LG}_{\ell, p}\left(\rho, \phi, z ; k_{0}\right) e^{i k_{0} z}$ (see Ref. [31]), where $k_{0}=2 \pi / \lambda_{0}$, and the indexes $\ell$ and $p$ correspond to the topological charge and the number of nonaxial radial nodes of the mode, respectively.

In Fig. 2(a), we show the intensity profile of the 21 st harmonic, resulting from the interaction with a $\lambda_{0}=0.8 \mu \mathrm{m}$ linearly polarized driving pulse, with a $\sin ^{2}$ envelope of 5.8 cycles $(15.4$ fs $)$ FWHM and peak intensity $I_{0}=1.4 \times 10^{14} \mathrm{~W} / \mathrm{cm}^{2}$. The driving field amplitudes ( $E_{0}$ of 0.075 and 0.087 a.u.) and waists ( $w_{0}$ of 30 and $21.4 \mu \mathrm{m}$ ) of the $\mathrm{LG}_{1,0}$ and $\mathrm{LG}_{2,0}$ modes, respectively, are chosen to give the same maximum intensity at the same radius for each mode at the focal plane, as depicted in Fig. 1(b). An argon gas jet is aimed perpendicularly to the IR laser and modeled with a transverse Gaussian profile (width $50 \mu \mathrm{m}$ ). Our HHG calculations, including propagation [37], are based on the numerical integration of the time-dependent Schrödinger equation using the strong-field approximation (SFA) at each atom out of a random sample of $>10^{5}$ atoms, distributed in the interaction region. In contrast to semiclassical SFA approaches, our method [38] retains the full quantum description of the interaction. The harmonic field radiated by each atom is propagated to the far-field detector using the integral solution of Maxwell equations. Details of this method are given in our previous studies of OAM HHG [19,39].

The upper panel in Fig. 2(a) represents the far-field spatial intensity distribution of the 21st harmonic as a function of the divergence $\beta$ and azimuth $\varphi$. The lower panel shows the OAM spectrum as a function of the divergence $\beta$, obtained after performing the Fourier transform of the harmonic field along $\varphi$. Note that these are interpolated pictures; the OAM must be interpreted in

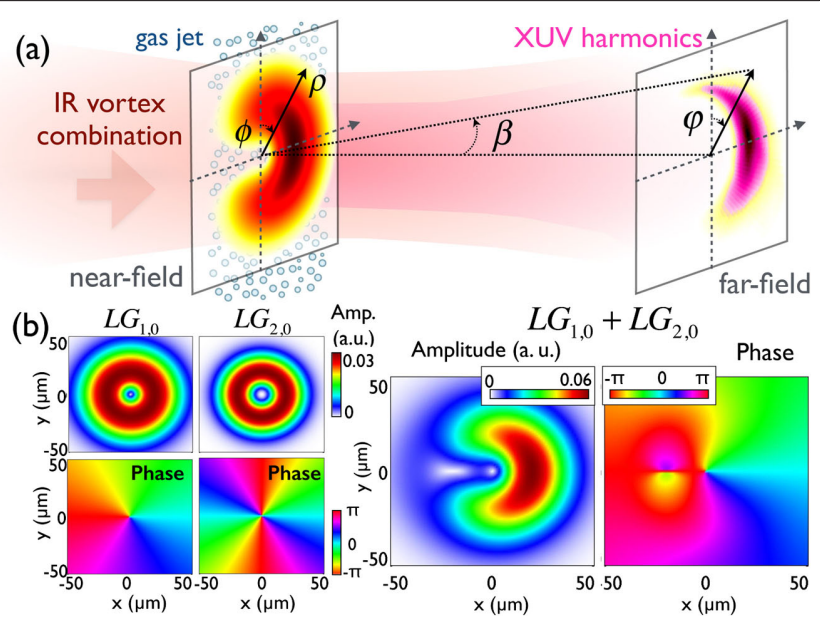

FIG. 1. (a) Schematic view of HHG driven by a combination of two vortex beams. An intense IR OAM pulse is focused into a gas jet with transverse coordinates $(\rho, \phi)$, where each atom emits harmonic radiation that, upon propagation, results in the far-field emission of XUV vortices with some divergence and azimuth $(\beta, \varphi)$. (b) The IR driving beam is composed by a combination of $\mathrm{LG}_{1,0}$ and $\mathrm{LG}_{2,0}$, with parameters chosen so the amplitude and radii at the ring maximum are the same.

discrete units. In contrast to HHG driven by a pure OAM vortex of charge $\ell[19,39]$, where each harmonic has a well-defined topological charge $\ell_{q}=q \ell$, the superposition of two vortices leads to a very rich composition of OAM for each harmonic order. As we discuss below, the nonperturbative character of $\mathrm{HHG}$ is the main thing responsible for the generation of new OAM contributions.

We have previously resorted to a thin slab model (TSM) [39] in order to gain insight on the physics of XUV vortices generated via HHG. In this simplified description, the target is represented by a thin (2D) slab perpendicular to the laser direction, located at the position of the gas jet. The harmonic field at the slab is approximated by an analytic SFA representation, first used in Ref. [40]. In this representation, the amplitude of the $q$ th harmonic is proportional to the $p$ th power of the fundamental field amplitude $U(\mathbf{r})^{p}$, where $p<q$ reflects the nonperturbative behavior of HHG, being approximately constant for the harmonics in the plateau region. In our case, we use $p=3.4$ (see Ref. [31]). On the other hand, the phase of the $q$ th harmonic is known to scale as $q$ times the phase of the driving field $q \Phi(\mathbf{r})$ and to have an additional term (intrinsic phase) $\alpha_{q}^{j}|U(\mathbf{r})|^{2}$, where $\alpha_{q}^{j}$ are strong-field parameters that depend on the electron quantum path (j) [41-43]. The intrinsic phase has a main role in HHG and phase matching. As it is well known, every half cycle, two different electron quantum paths contribute to the same harmonic, the so-called short and long trajectories $[41,44]$. The contribution to HHG of each trajectory type is mainly through the different intrinsic phases, described by the coefficients $\alpha_{q}^{j}$. In particular, the $j$ quantum path 

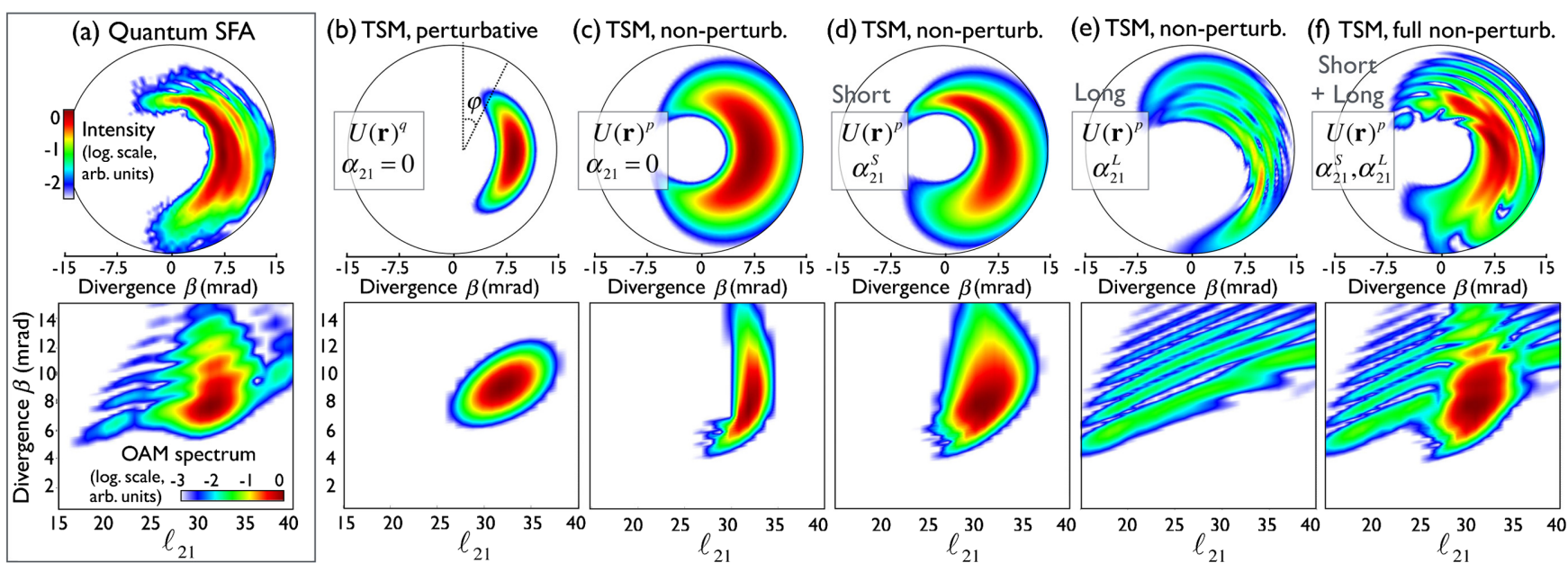

FIG. 2. Nonperturbative OAM generation in the 21st harmonic vortex beam. The upper row represents the far-field spatial intensity distribution (logarithmic scale), and the lower row shows the OAM spectrum (logarithmic scale). The results were obtained through (a) quantum SFA simulations and (b)-(f) different versions of the TSM, where we have considered (b) perturbative ( $q$ th power, no intrinsic phase), (c) nonperturbative $p$ th power (no intrinsic phase), and full nonperturbative (intrinsic phase included) with (d) short, (e) long, and (f) short + long trajectory contributions. The excellent agreement between (a) and (f) supports the use of the TSM to understand the nonperturbative OAM contributions in the quantum calculation.

(short or long) to the $q$ th harmonic emitted at a slab located at the beam focus $(z=0)$ can be written as

$$
A_{q}^{j}(\rho, \phi)=\left(\frac{C}{\tau^{j}}\right)^{3 / 2}|U(\rho, \phi)|^{p} e^{i q \Phi(\rho, \phi)} e^{i \alpha_{q}^{j}|U(\rho, \phi)|^{2}}
$$

where $C$ is a constant and $\tau^{j}$ is the excursion time associated with the $j$ quantum path [45], affecting the efficiency of $\mathrm{HHG}$ due to the quantum diffusion of the electron wave packet. In our case, $U(\mathbf{r})=$ $\mathrm{LG}_{1,0}\left(\mathbf{r}, k_{0}\right)+\mathrm{LG}_{2,0}\left(\mathbf{r}, k_{0}\right)$, as depicted in Fig. 1(b). Our TSM approach evaluates the far-field harmonic integrating $A_{q}^{j}(\rho, \phi)$ over the slab surface, using the Fraunhofer diffraction formula

$F_{q}^{j}(\beta, \varphi) \propto \int_{0}^{\infty} \int_{0}^{2 \pi} \rho d \rho d \phi A_{q}^{j}(\rho, \phi) e^{-i q k_{0} \rho \tan \beta \cos (\varphi-\phi)}$.

The TSM neglects the longitudinal phase matching, thus focusing the discussion on the transverse phase matching [46], which is then relevant, due to the unique transverse structure of OAM beams [39]. Figures 2(b)-2(f) show the TSM results in comparison with the quantum SFA simulations discussed above. In order to disentangle the nonperturbative effects in OAM HHG, we first show the results obtained as if the process was perturbative; i.e., the intrinsic phase is neglected $\left(\alpha_{21}^{j}=0\right)$ and the amplitude of the harmonic field scales with the $q$ th power $(p=q=21)$ [Fig. 2(b)]. Note that even in the perturbative case, the OAM spectra of the harmonic radiation is already more involved than using a pure vortex. This can be understood as different photon absorption channels $(q-n, n), n$ ranging from 0 to $q$, associated with the two OAM modes of the driving field. Note that this interpretation in terms of absorption channels is analogous to that performed when driving HHG by noncollinear [47-49] or by bicircular fields [50]. Each channel $(q-n, n)$ has a weight proportional to the binomial probability distribution associated with the photon number combinations of absorbing $q-n$ photons from mode $\ell_{1}$ and $n$ photons from mode $\ell_{2}$. Thus, the perturbative OAM conservation rule reads as $\ell_{q}=(q-n) \ell_{1}+n \ell_{2}$.

Figure 2(c) shows the role of the nonperturbative $p$ th power scaling by using $p=3.4<q$ (the intrinsic phase remains neglected $\alpha_{21}^{j}=0$ ). Now, the OAM spectra narrows around the central channel, $n$ ranging from $(q-p) / 2$ to $(q+p) / 2$. This channel suppression is further analyzed in Fig. 3(a), where we show the $\beta$-integrated OAM spectrum of the 21st harmonic using the $q$ th power (dark blue) and the $p$ th power (light blue, $p=3.4$ ) harmonic scaling. A nonperturbative channel reduction towards channels $n=9\left(\ell_{21}=30\right)$ to $n=12\left(\ell_{21}=33\right)$ is observed.

Figures 2(d) and 2(e) show the result of the TSM, including the $p$ th power scaling and the intrinsic phase term, for the short and long trajectories (respectively), leading to the 21st harmonic. We use $\alpha_{21}^{S}=3.83 \times 10^{-14} \mathrm{~cm}^{2} / \mathrm{W}$ and $\alpha_{21}^{L}=2.128 \times 10^{-13} \mathrm{~cm}^{2} / \mathrm{W}$ for the short and long trajectories, respectively [31]. First, we note that long trajectories present a more irregular radial profile than the short ones, as already mentioned in Ref. [39]. Second, in both cases, and especially in the later one, we observe a dramatic broadening of the OAM spectrum. According to Eq. (2), the spatial variations in the driving field intensity profile are imprinted as local modifications of the intrinsic phase of the harmonics. For periodic azimuthal variations of the intensity, as those arising from the superposition of different OAM beams [see Fig. 1(b)], the situation is analogous to applying a phase 


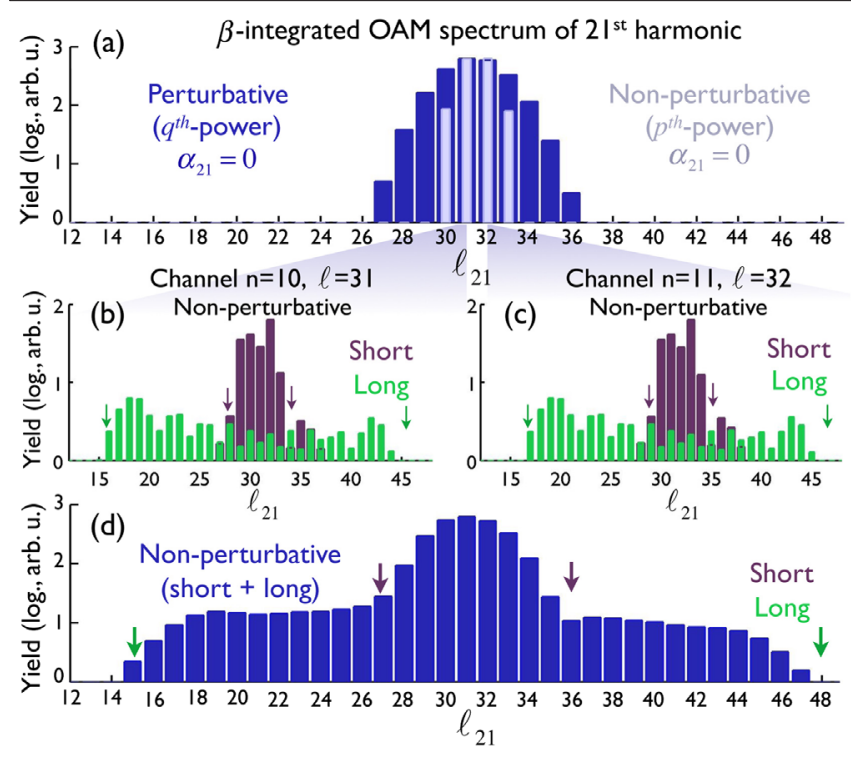

FIG. 3. $\beta$-integrated OAM spectrum (logarithmic scale) of the 21st harmonic vortex, obtained with the TSM using (a) the perturbative $q$ th power [dark blue, Fig. 2(b)] and the nonperturbative $p$ th power scaling [light blue, Fig. 2(c)]; (b),(c) nonperturbative channels $n=10$ and $n=11$, respectively, including the intrinsic phase of short (purple) and long (green) trajectories; and (d) all nonperturbative channel contributions [corresponding to Fig. 2(f)]. The arrows indicate the maximum OAM interval given by the conservation rules for the short (purple) and long (green) trajectory contributions.

diffraction grating along the azimuthal coordinate. Thus, new OAM contributions are produced, as phase diffraction gratings produce multiple diffraction orders. The nonperturbative OAM broadening is shown in detail in Figs. 3(b) and 3(c) for the $n=10$ and $n=11$ channels, respectively, and for the short (purple) and long (green) trajectory contributions. The OAM content of each channel is dramatically increased due to the intrinsic phase, from a single to a wide range of OAM values.

Figure 2(f) shows the complete nonperturbative profile for the 21st harmonic, resulting from the superposition of the short and long trajectory contributions. The excellent agreement between Figs. 2(f) and 2(a) confirms the validity of the TSM. The short and long trajectory contributions can be disentangled in the quantum SFA calculations through time-frequency analysis (see Ref. [31]).

The azimuthal integration in Eq. (2) leads to the derivation of a general conservation rule for the nonperturbative OAM buildup $\left(\ell_{q}\right)$ of the $q$ th harmonic (see details in Ref. [31]):

$$
\ell_{q}=(q-n) \ell_{1}+n \ell_{2}+m\left(\ell_{2}-\ell_{1}\right)
$$

$n$ labeling the HHG channel [ranging from $(q-p) / 2$ to $(q+p) / 2]$. The index $m$ is limited approximately by $|m|<\alpha_{q}^{j} I_{0}(\rho) / 2$ (see Ref. [31]). It represents the azimuthal diffraction order induced by the intrinsic phase modulation, which has an upper bound, or cutoff, given by the maximum driving field intensity at each particular radius $I_{0}(\rho)$. Note that this conservation rule collapses to the perturbative case when $p \rightarrow q$ and $m=0$, and to the linear scaling found with pure vortex drivings when $\ell_{1}=\ell_{2}=\ell[19,20]$. Figure 3(d) shows the full nonperturbative, $\beta$-integrated, OAM spectrum of the 21st harmonic. The arrows indicate the maximum OAM intervals given by Eq. (3) for the short (purple) and long (green) trajectory contributions, validating the nonperturbative OAM conservation rules obtained. Additional results for other harmonic orders and vortex combinations are presented in Ref. [31].

OAM contributions of a complex vortex beam can be successfully separated in the visible region $[51,52]$. The rich OAM composition of the harmonic vortices presented here may stimulate the extension of such techniques to the XUV region, opening a fascinating scenario for the production of XUV OAM tunable beams directly from the HHG process.

We finally address the scenario of a nonpure vortex driver as a realistic approach to an experiment. In OAM HHG [18,20,21], and also in nonlinear propagation experiments $[53,54]$, the intense driver vortex beam might present imperfections, arising from the mechanism used to generate ultrashort, broadband vortex beams (typically, wave plates or diffractive elements are used [55-57]). In fact, in the nonlinear propagation studies, such imperfections must be introduced in the theoretical models to mimic the experimental observations [53,54]. To study the effect of these imperfections in OAM HHG, we compare the OAM harmonic spectrum driven by a pure $\mathrm{LG}_{1,0}$ mode [Fig. 4(a)] and quasipure $\mathrm{LG}_{1,0}$ modes [Figs. 4(b) and 4(c)] (having a
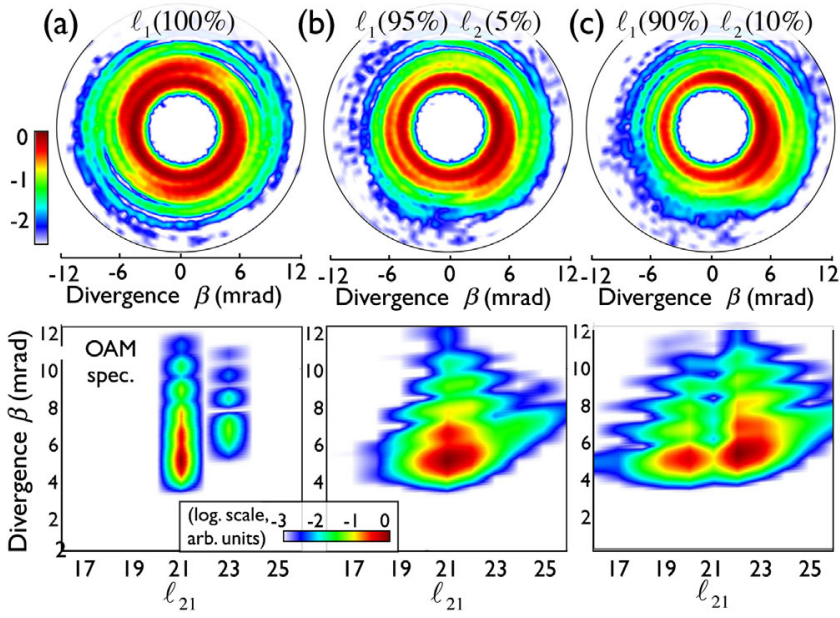

FIG. 4. Quantum SFA simulations of the 21st-harmonic vortex structure driven by (a) a pure $\ell=1$ mode, and nonpure OAM beams, with combination of (b) $95 \% \ell_{1}=1$ and $5 \% \ell_{1}=2$, and (c) $90 \% \ell_{1}=1$ and $10 \% \ell_{1}=2$. The beam waists are the same as in Fig. 1b. The top row represents the harmonic intensity profiles, which are found to be similar. In the bottom row, the OAM spectra of 21 st-harmonic are shown, presenting a clear broadening around the single-vortex value $\ell_{21}=21$ from (a) to (c). 
contribution of a $5 \%$ and $10 \%$ of an $\mathrm{LG}_{2,0}$ mode, respectively). Surprisingly, although the spatial intensity distribution is similar, the nonpure cases present a clear broadening of the 21st harmonic OAM content around the single-vortex value $\ell_{21}=21$. Interestingly, in Fig. 4(c), the efficiency of this channel is diminished in favor of the mixture of 20 photons of the $\ell_{1}$ mode and 1 photon of $\ell_{2}$, accordingly to the weights given by the binomial probability distribution. We, therefore, demonstrate that the nonperturbative nature of OAM HHG is also relevant when dealing with quasipure vortices, which is a common experimental situation. Our results open the route to use the generated OAM spectra in HHG as an additional tool to characterize ultrashort optical vortex pulses [58].

In conclusion, we demonstrate that the nonperturbative nature of $\mathrm{HHG}$ encloses a rich scenario for the generation of XUV vortices. We derive the conservation rule for the OAM buildup, which predicts a strong twist of the XUV vortices due mainly to the intrinsic HHG phase. Our results show that the laser-matter coupling with OAM beams is a very sensitive probe of the nonperturbative aspects of strong-field interactions, paving the route for the next generation of high-resolution XUV or x-ray diagnostic tools for fundamental studies and applications. Our model predictions demonstrate the correlation of the generated XUV vortex beams with the driving field parameters. Therefore, HHG can be used as a controlled, straightforward mechanism for the generation of complex vortex beams at different wavelengths.

C. H.-G. acknowledges support by a Marie Curie International Outgoing Fellowship within the EU Seventh Framework Programme for Research and Technological Development (2007-2013), under REA Grant Agreement No. 328334. We acknowledge support from Junta de Castilla y León (Projects No. SA116U13 and No. SA046U16) and Ministerio de Economía y Competitividad (FIS2013-44174P, FIS2016-75652-P). A. P. acknowledges support from the U.S. Department of Energy, Office of Science, Basic Energy Sciences, Chemical Sciences, Geosciences, and Biosciences Division under Contract No. DE-AC02$06 \mathrm{CH} 11357$.

*laura.rego@usal.es

†carloshergar@usal.es

[1] P. Agostini and L. F. DiMauro, Rep. Prog. Phys. 67, 813 (2004).

[2] F. Krausz and M. Ivanov, Rev. Mod. Phys. 81, 163 (2009).

[3] J. Miao, T. Ishikawa, I. K. Robinson, and M. M. Murnane, Science 348, 530 (2015).

[4] K. J. Schafer, B. Yang, L. F. DiMauro, and K. C. Kulander, Phys. Rev. Lett. 70, 1599 (1993).

[5] P. B. Corkum, Phys. Rev. Lett. 71, 1994 (1993).

[6] M. B. Gaarde, J. L. Tate, and K. J. Schafer, J. Phys. B 41, 132001 (2008).
[7] T. Popmintchev, M. Chen, P. Arpin, M. M. Murnane, and H. C. Kapteyn, Nat. Photonics 4, 822 (2010).

[8] L. A. Lompré, A. L'Huillier, M. Ferray, P. Monot, G. Mainfray, and C. Manus, J. Opt. Soc. Am. B 7, 754 (1990).

[9] T. Popmintchev, M.-C. Chen, D. Popmintchev, P. Arpin, S. Brown, S. Alisauskas, G. Andriukaitas, T. Balciunas, O. Mücke, A. Pugzlys, A. Baltuska, B. Shim, S. E. Schrauth, A. Gaeta, C. Hernández-García, L. Plaja, A. Becker, A. Jaroń-Becker, M. M. Murnane, and H. C. Kapteyn, Science 336, 1287 (2012).

[10] D. Popmintchev et al., Science 350, 1225 (2015).

[11] G. Farkas and C. Toth, Phys. Lett. A 168, 447 (1992).

[12] I. P. Christov, M. M. Murnane, and H. C. Kapteyn, Phys. Rev. Lett. 78, 1251 (1997).

[13] P. M. Paul, E. S. Toma, P. Breger, G. Mullot, F. Augé, Ph. Balcou, H. G. Muller, and P. Agostini, Science 292, 1689 (2001).

[14] M. Hentschel, R. Kienberger, C. Spielmann, G. A. Reider, N. Milosevic, U. Heinzmann, M. Drescher, and F. Krausz, Nature (London) 414, 509 (2001).

[15] P. B. Corkum and F. Krausz, Nat. Phys. 3, 381 (2007).

[16] P. Salières, A. Maquet, S. Haessler, J. Caillat, and R. Taïeb, Rep. Prog. Phys. 75, 062401 (2012).

[17] S. Baker, J. S. Robinson, C. A. Haworth, H. Teng, R. A. Smith, C. C. Chirila, M. Lein, J. W. G. Tisch, and J.P. Marangos, Science 312, 424 (2006).

[18] M. Zürch, C. Kern, P. Hansinger, A. Dreischuh, and Ch. Spielmann, Nat. Phys. 8, 743 (2012).

[19] C. Hernández-García, A. Picón, J. San Román, and L. Plaja, Phys. Rev. Lett. 111, 083602 (2013).

[20] G. Gariepy, J. Leach, K. T. Kim, T. J. Hammond, E. Frumker, R. W. Boyd, and P. B. Corkum, Phys. Rev. Lett. 113, 153901 (2014).

[21] R. Géneaux, A. Camper, T. Auguste, O. Gobert, J. Caillat, R. Taeib, and T. Ruchon, Nat. Commun. 7, 12583 (2016).

[22] L. Allen, M. W. Beijersbergen, R. J. C. Spreeuw, and J. P. Woerdman, Phys. Rev. A 45, 8185 (1992).

[23] G. F. Calvo, A. Picón, and E. Bagan, Phys. Rev. A 73, 013805 (2006).

[24] M. S. Soskin and M. V. Vasnetsov, Prog. Opt. 42, 219 (2001).

[25] Twisted Photons, edited by J.P. Torres and L. Torner (Wiley-VCH, Weinheim, 2011).

[26] A. Picón, A. Benseny, J. Mompart, J. R. Vázquez de Aldana, L. Plaja, G. F. Calvo, and L. Roso, New J. Phys. 12, 083053 (2010).

[27] I. V. Basistiy, V. Yu. Bazhenov, M. S. Soskin, and M. V. Vasnetsov, Opt. Commun. 103, 422 (1993).

[28] K. Dholakia, N. B. Simpson, M. J. Padgett, and L. Allen, Phys. Rev. A 54, R3742 (1996).

[29] J. Courtial, K. Dholakia, L. Allen, and M. J. Padgett, Phys. Rev. A 56, 4193 (1997).

[30] A. Berzanskis, A. Matijosius, A. Piskarskas, V. Smilgevicius, and A. Stabinis, Opt. Commun. 140, 273 (1997).

[31] See Supplemental Material at http://link.aps.org/ supplemental/10.1103/PhysRevLett.117.163202 for a detailed discussion of (i) the definition of the Laguerre Gaussian modes, (ii) the scaling of the harmonic amplitude with the driver field, (iii) the derivation of the OAM conservation rules, (iv) the quantum-path identification through time-frequency analysis, and (v) additional results 
for other harmonic orders and other driving OAM combinations. It includes Refs. [32-36].

[32] L. V. Keldysh, Zh. Eksp. Teor. Fiz. 47, 1945 (1964) [Sov. Phys. JETP 20, 1307 (1965)].

[33] F. H. M. Faisal, J. Phys. B 6, L89 (1973).

[34] H. R. Reiss, Phys. Rev. A 22, 1786 (1980).

[35] J. A. Pérez-Hernández, L. Roso, and L. Plaja, Opt. Express 17, 9891 (2009).

[36] Y. Mairesse, A. de Bohan, L. J. Frasinski, H. Merdji, L. C. Dinu, P. Monchicourt, P. Breger, M. Kovačev, R. Taïeb, B. Carré, H. G. Muller, P. Agostini, and P. Saliéres, Science 302, 1540 (2003).

[37] C. Hernández-García, J. A. Pérez-Hernández, J. Ramos, E. Conejero Jarque, L. Roso, and L. Plaja, Phys. Rev. A 82, 033432 (2010).

[38] J. A. Pérez-Hernández, C. Hernández-García, J. Ramos, E. Conejero Jarque, L. Plaja, and L. Roso, New Methods For Computing High-Order Harmonic Generation and Propagation, Springer Series in Chemical Physics, Vol. 100 (Springer, New York, 2011), Chap. 7, p. 145.

[39] C. Hernández-García, J. San Román, L. Plaja, and A. Picón, New J. Phys. 17, 093029 (2015).

[40] A. L'Huillier, Ph. Balcou, S. Candel, K. J. Schafer, and K. C. Kulander, Phys. Rev. A 46, 2778 (1992).

[41] M. Lewenstein, P. Salieres, and A. L'Huillier, Phys. Rev. A 52, 4747 (1995).

[42] A. Zaïr, M. Holler, A. Guandalini, F. Schapper, J. Biegert, L. Gallmann, U. Keller, A. S. Wyatt, A. Monmayrant, I. A. Walmsley, E. Cormier, T. Auguste, J. P. Caumes, and P. Salières, Phys. Rev. Lett. 100, 143902 (2008).

[43] C. Hernández-García and L. Plaja, J. Phys. B 45, 074021 (2012).

[44] M. Bellini, C. Lynga, A. Tozzi, M. B. Gaarde, T. W. Hänsch, A. L'Huillier, and C. G. Wahlström, Phys. Rev. Lett. 81, 297 (1998).
[45] M. Lewenstein, Ph. Balcou, M. Yu. Ivanov, A. L'Huillier, and P. B. Corkum, Phys. Rev. A 49, 2117 (1994).

[46] C. Hernández-García, I. J. Sola, and L. Plaja, Phys. Rev. A 88, 043848 (2013).

[47] A. V. Birulin, V. T. Platonenko, and V. V. Strelkov, Quantum Electron. 26, 377 (1996).

[48] J. B. Bertrand, H. J. Wörner, H. C. Bandulet, É. Bisson, M. Spanner, J.-C. Kieffer, D. M. Villeneuve, and P. B. Corkum, Phys. Rev. Lett. 106, 023001 (2011).

[49] D. D. Hickstein, F. J. Dollar, P. Grychtol, J. L. Ellis, R. Knut, C. Hernández-García, C. Gentry, D. Zusin, J. M. Shaw, T. Fan, K. M. Dorney, A. Becker, A. Jaroń-Becker, H. C. Kapteyn, M. M. Murnane, and Ch. G. Durfee, Nat. Photonics 9, 743 (2015).

[50] A. Fleischer, O. Kfir, T. Diskin, P. Sidorenko, and O. Cohen, Nat. Photonics 8, 543 (2014).

[51] G. C. G. Berkhout, M. P. J. Lavery, J. Courtial, M. W. Beijersbergen, and M. J. Padgett, Phys. Rev. Lett. 105, 153601 (2010).

[52] M. Mirhosseini, M. Malik, Z. Shi, and R. W. Boyd, Nat. Commun. 4, 2781 (2013).

[53] L. T. Vuong, T. D. Grow, A. Ishaaya, A. L. Gaeta, G. W. 't Hooft, E. R. Eliel, and G. Fibich, Phys. Rev. Lett. 96, 133901 (2006).

[54] A. A. Ishaaya, L. T. Vuong, T. D. Grow, and A. L. Gaeta, Opt. Lett. 33, 13 (2008).

[55] I. J. Sola, V. Collados, L. Plaja, C. Méndez, J. San Román, C. Ruiz, I. Arias, A. Villamarín, J. Atencia, M. Quintanilla, and L. Roso, Appl. Phys. B 91, 115 (2008).

[56] K. Yamane, Y. Toda, and R. Morita, Opt. Express 20, 18986 (2012).

[57] J. Atencia, M. V. Collados, M. Quintanilla, J. Marin-Saez, and I. J. Sola, Opt. Express 21, 21056 (2013).

[58] M. Miranda, M. Kotur, P. Rudawski, C. Guo, A. Harth, A. L'Huillier, and C. L. Arnold, arXiv:1505.06081. 